\title{
Applications of Free Electron Lasers in Biology and Medicine
}

\author{
J.B. PeŁkA ${ }^{a, *}$, K.R. Tybor ${ }^{b}$, R. NietubyĆ ${ }^{c}$ And G. Wrochna ${ }^{c}$ \\ ${ }^{a}$ Institute of Physics, Polish Academy of Sciences, al. Lotników 32/46, 02-668 Warsaw, Poland \\ ${ }^{b}$ Department of Neurosurgery, Medical University of Łódź, Łódź, Poland \\ ${ }^{c}$ The Andrzej Soltan Institute for Nuclear Studies, Świerk, Poland
}

\begin{abstract}
The advent of free electron lasers opens up new opportunities to probe the dynamics of ultrafast processes and the structure of matter with unprecedented spatial and temporal resolution. New methods inaccessible with other known types of radiation sources can be developed, resulting in a breakthrough in deep understanding the fundamentals of life as well as in numerous medical and biological applications. In the present work the properties of free electron laser radiation that make the sources excellent for probing biological matter at an arbitrary wavelength, in a wide range of intensities and pulse durations are briefly discussed. A number of biophysical and biomedical applications of the new sources, currently considered among the most promising in the field, are presented.
\end{abstract}

PACS numbers: 87.59.-e, 41.60.Cr, 07.85.Qe, 82.53.Ps, 87.64.Bx

\section{Introduction}

Classic optical lasers generate beams that are constrained to photon energies determined by discrete quantum states in an atomic gain medium. Contrary to this, the free electron lasers (FELs) utilize a relativistic electron beam from an accelerator as a lasing medium. The monochromatic radiation is generated when the accelerated beam passes through a periodic structure of alternating in-line magnetic field formed in undulator. The wavelength of the emitted radiation depends only on the undulator magnetic period, the intensity of magnetic field in the undulator, and the energy of accelerated electrons that can be continuously tuned to arbitrary values. Apart easy tunability over a reasonable photon energy range, the radiation is characterized with a unique combination of coherence, polarization, and high power/pulse. Its complex superpulse structure met in some machines, as well as ability to operate in various modes of pulse emission, from quasicontinuous up to ultrafast, several femtosecond single pulses is also advantageous in many applications. With these emission properties FELs span gap between conventional lasers and synchrotron radiation (SR) sources.

Though all free electron lasers consist of an electron accelerator and an undulator as the major parts, two main types should be distinguished. The one, known as oscillator FEL or low-gain FEL operates in a multipass mode. This means that, due to a low gain, the generated light beam is accumulated in subsequent acts of interaction of the electromagnetic energy trapped in an optical cavity with electron bunches within the cavity. This type

* corresponding author; e-mail: pelkay@ifpan.edu.pl of FEL has, similarly to classical lasers, a lower wavelength limit of operation due to cavity construction limitations. Contrary to this, high-gain FELs do not need cavity to operate, getting all the emission energy in a single pass through a long undulator with a single electron bunch. The two types of free electron lasers are compared in Figs. 1 and 2, where schematic views of the low-gain free electron laser with optical cavity (Fig. 1) and of the high gain self amplified spontaneous emission FEL (SASE-FEL) with superconducting electron accelerator modules (Fig. 2) are shown. A concise review of FEL principles and types can be found elsewhere (see, e.g. a tutorial review on free electron laseres by Khan [1] and references cited therein).

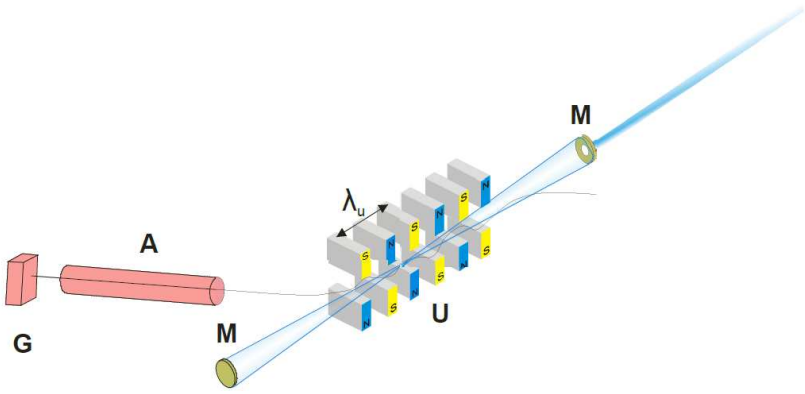

Fig. 1. Schematic view of low-gain free electron laser with optical cavity. G - electron gun, A - accelerator, $\mathrm{U}$ - undulator, $\mathrm{M}$ - mirrors.

First FELs started operation since 1977, delivering radiation at wavelengths in the mid-IR region of few $\mu \mathrm{m}$. Up to 1988 the shortest attainable wavelength was $240 \mathrm{~nm}$ with a small progress to $212 \mathrm{~nm}$ ten years later. By the end of 20th century the lowest wavelength moved 


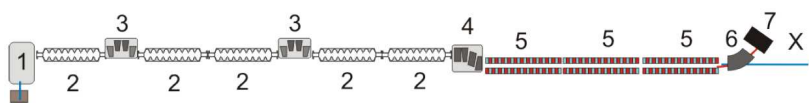

Fig. 2. Schematic view of high-gain SASE FEL with superconducting electron accelerator modules. 1 electron gun with laser-driven photocatode, $2-\mathrm{SC}$ modules of electron accelerator, 3 - electron beam compressor (chicane), 4 - electron beam collimator, 5 undulator sections, 6 - bending magnet, 7 - electron beam absorber, $\mathrm{X}$ - laser beam.

down to $193.7 \mathrm{~nm}$, all this with optical cavity, low-gain FELs [2]. With the introduction, at the turn of centuries, of high-gain SASE FEL, capable of delivering radiation without any optical cavity, the limit has moved rapidly to $100-120 \mathrm{~nm}$ in 1999 . In the next decade the minimal wavelength has been shifted up to $6.3 \mathrm{~nm}$ at FLASH free electron laser user facility in Hamburg [3]. High-gain FELs, contrary to other laser sources, can operate in the spectral regions of short wavelengths, ranging from extreme UV through soft and hard X-rays, with peak power that can exceed more than $1 \mathrm{GW}$ in a single ultrafast pulse of 10-20 fs and less. Spectral brightness of the new X-FEL sources under construction, to be operational in the next few years, will be up to 9 orders of magnitude higher, as compared to the most intense 3rd generation light sources and will reach a value of $10^{29}$ photons/( $\left.\mathrm{s} \mathrm{mrad}^{2} \mathrm{~mm}^{2} 0.1 \% \mathrm{BW}\right)$, emitted in ultrafast pulses of less than 10 fs [4]. Due to mentioned above properties, as well as simple structure of ultrafast pulses, the SASE FELs have been recognized as excellent sources to probe the dynamics of ultrafast processes and the structure of matter with unprecedented spatial and temporal resolution, making it possible to apply methods inaccessible with other known types of radiation sources.

Following the success of SR sources, extensively applied in biology and in medicine for more than thirty years [5-7], intense FEL beams opened up new perspectives. The new type of source was found soon to be excellent for probing soft tissue with superior sensitivity, in a wide range of wavelengths, intensities and pulse durations, capable to detect even small tissue variations. A rapidly growing interest in application of FEL beams is observed in many areas of biomedical science opening up new opportunities to study life forms on different levels, from biomolecules and subcellular structures up to whole organisms.

The aim of this work is to draw some exemplary applications at FEL sources operating with various ranges of wavelengths that are at present recognized among the most promising to the development of biomedical science and new medical techniques. Most of the described techniques are anticipated to be included in the scientific program at the Polish Free Electron Laser (POLFEL), proposed to be built at the Andrzej Sołtan Institute for Nuclear Studies in Swierk near Warsaw [8].

\section{FEL for surgery}

Interaction of intense laser beams with tissue has been systematically investigated soon after FEL invention. The new light sources contributed mainly with their easy tunability and time structure of pulsed emission to a stormy development of biomedical laser techniques. Due to this, FELs turned out to be superior to regularly used classical laser sources in optimizing the wavelength-dose-pulse structure pattern to various types of tissue ablation and in search for minimizing the adverse effects in photoablative surgical techniques [9].

The optimal wavelength at which laser ablates a tissue depends primarily on the relative (differential) absorption of main tissue components, that is the water, the mineral components, if any, and the specific proteins. Thus, knowing the absorption spectrum of a tissue, some wavelengths can be guessed to be potentially suitable for optimal ablation (characterized by a high efficiency and low collateral damage). However, a complexity of photoablation processes makes it necessary to experimentally confirm the parameters optimal for the ablation of a specific tissue.

Most of this work has been done in the mid-IR range of wavelengths, partially due to the fact that early low-gain FELs delivered mostly a long-wavelength radiation. Few characteristic absorption bands have been identified as potentially suitable to optimal ablation in the range of $1-10 \mu \mathrm{m}$. In this water (OH stretch mode), $2.94 \mu \mathrm{m}$, protein (amide-II band), $6.45 \mu \mathrm{m}$, both water $(\mathrm{H}-\mathrm{O}-\mathrm{H}$ bend) and protein (amide-I band), 6.10-6.12 $\mu \mathrm{m}, 7.01 \mu \mathrm{m}(\mathrm{C}-\mathrm{O}$ stretch of cholesterol), $8.17 \mu \mathrm{m}$ ( $\mathrm{P}-\mathrm{O}-\mathrm{P}$ antisymmetric stretch $), 8.525 \mu \mathrm{m}(\mathrm{C}=\mathrm{O}$ stretch of protein). A band at $2.79 \mu \mathrm{m}$ associated with the potential shift in the dynamic optical properties of water during tissue irradiance with the intense laser beam was also considered [10].

In a number of experiments with IR-FEL sources, the surgical ablation of specific hard and soft tissues was studied at various wavelengths and in many cases the optimal conditions were determined. For the cortical bone ablation, the highest mass removal occurred at $6.1 \mu \mathrm{m}$, where also the least collateral thermal injury was produced $[9,10]$. The surface modification of root dentin was found to be strongly dependent on the laser parameters applied. The optimum wavelengths for laser treatment of root surface caries were estimated to $9.0 \mu \mathrm{m}$ or near to $9.7 \mu \mathrm{m}$, corresponding to the absorption peak due to $\mathrm{P}-\mathrm{O}$ stretching in the root dentin [11]. Selective removal of cholesterol ester in carotid artery atheromatous plaques with FEL emitting radiation at $5.75 \mu \mathrm{m}$ wavelength was applied to treat intimal slices of extirpated human arterial atherosclerotic plaques [12]. It was demonstrated that, differently from previous approaches involving conventional lasers, FEL irradiation can remove cholesterol ester selectively from human atheromatous CA plaques.

Cornea was treated with FEL tuned to $6 \mu \mathrm{m}$ range. Larger etch depths were found for larger spot sizes - an effect that can lead to an apparent wavelength dependence. Plume imaging at several wavelengths and spot 
sizes suggested that this effect is due to increased post-pulse ablation at larger spots [13]. In a similar way, basing on evaluation and comparison of absorption spectra of fat and water, free electron laser was applied to investigate the selective photothermolysis of lipid-rich tissue. Exploiting the wavelengts between $1.21 \mu \mathrm{m}$ and $1.72 \mu \mathrm{m}$, where fat absorbs radiation more than water, there was demonstrated a selective damage of fat without damage to overlying epidermis and dermis (with additional cooling during ablation) [14].

Investigations with a Mark-III free electron laser at Vanderbildt University, tuned to $6.45 \mu \mathrm{m}$ in wavelength have demonstrated minimal collateral damage and high ablation yield in ocular and neural tissues. The FEL energy at this wavelength delivered through a hollow waveguide appears capable of efficiently and safely producing an optic nerve sheath fenestration in monkeys. The authors suggested the innovative surgical technique to be considered for human use [15]. The experiments on brain ablation in the rat cerebral cortex have confirmed that wavelengths in the mid-IR could be selected for optimal ablative properties [16].

Impact on cells outside the lethal zone during the ablation of skin was also investigated in some experiments. In a recent work, Wilmink et al. [17] applied the heat shock protein (HSP) expression as a sensitive quantitative marker of sublethal damage in a transgenic mouse strain. The magnitude of hsp70 expression was found to be dose-dependent and maximal 5 to $12 \mathrm{~h}$ after surgery. The results of the experiment suggested that the $6.10 \mu \mathrm{m}$ wavelength is a superior wavelength for laser ablation of skin.

A number of studies were devoted to investigate the ablation and damage mechanisms. Water thermodynamics is considered as the essential contributor to the overall mechanisms of laser-tissue interaction [18, 19]. Free-electron laser irradiation can superheat tissue water, driving thermal vapor bubbles confined by tissue matrix and leading to tissue ablation. For example, highly focused laser pulses in the near infrared (IR), visible, or near UV have been used to drive plasma formation and cavitation dynamics in water, both in bulk [20] and as confined by biological matrix [21]. Edwards and Hutson [22] discussed mechanisms of tissue ablation at $6.45 \mu \mathrm{m}$. Despite the fact that absorption band of the tissues at this wavelength is not the strongest in the mid-infrared, the FEL ablation is efficient while minimizing collateral damage. This wavelength dependence was explained as a competition between two dynamic processes - explosive vaporization of saline and denaturation of structural proteins. It was shown that the above model predicts for each wavelength a region of parameter space (incident intensity and pulse width) in which explosive vaporization is preceded by substantial protein denaturation. This region is much larger for wavelengths where protein is the dominant chromophore. At wavelengths for which this condition is not fulfilled, collateral damage may be minimized, but the maximum intensities and pulse widths in these regions are insufficient to remove tissue at surgically relevant rates.

Recent work by Wagner et al. [23] confirmed wavelength-dependent differences in the pressure dynamics associated with tissue ablation by a mid-IR FEL. For $3.0 \mu \mathrm{m}$ wavelength, the water bubble collapse was identified as a main process associated with tissue failure. Contrary to this, for $6.45 \mu \mathrm{m}$ irradiation, the failure was induced primarily during bubble growth.

Although many diverse issues can significantly profit of FEL beams, their direct use in surgery is limited due to a large scale and specific demands of these devices that cannot be easily adopted in clinical environment. To try out the real clinical abilities of the new sources in human medicine, an operating room was appended onto an FEL environment at Vanderbilt Keck FEL Center, instead of transplanting a whole FEL system into the hospital setting. The machine, delivering radiation in the mid-IR wavelength range, has been successfully used in human nuerosurgery, oncological surgery and ophthalmology [24-26].

Some surgery-oriented studies have been carried out also in the UV and deep-UV regions, using, e.g., OK-4/ Duke FEL based on storage ring with electron energies up to $750 \mathrm{MeV}$ that made it possible to reach wavelengths as low as $217 \mathrm{~nm}$ [27]. It should be however stressed that studies in the shorter range of wavelength are at their beginning. Irradiation with shorter wavelength leads to specific ablation mechanisms with a susbstantial increase in ionization radiation damage. It is due to the fact that quanta of higher energies are able to directly cut chemical bonds, as well as the wavelength-dependent absorption lengths differently affect the pulse energy deposition in a tissue.

The biggest obstacle with implementing the conventional optical lasers into neurosurgery was unforeseeable effect on surrounding structures, e.g. deep brain areas or cranial nerves. The other problem was the dependence of the laser effect on degree of tissue vasculature. Thus, in the regions of the brain with the denser (brain cortex) and thinner (white matter) vasculature, the laser ablation went differently. Finally, the effect of carbonisation of the neural tissue made laser action difficult and time wasting. The "ideal" laser as an instrument in neurosurgery needs to be a perfect, the most accurate, bloodless knife, or selective only to pathological tissue (e.g. tumor). Unfortunately, the most frequent brain tumors infiltrate vast operative brain regions, so it is not possible to remove the whole tumor in conventional manner. Free electron lasers are expected to give here a substantial contribution in detailed understanding the damage/ablation mechanisms and their dependence on FEL beam parameters like wavelength, fluence, and pulse temporal structure. As a consequence, new optimized technques and devices can be developed. 


\section{Bioimaging with FEL sources}

It is well known that the diffraction defines the principal limit for image resolution in optical systems. It is particularly inconvenient at longer wavelengths, when for the investigation of biological specimens it is advantageous to apply optical infrared microscopy with possibly high, submicrometric lateral resolution. Imaging below classical diffraction limit has been made possible with a scanning near-field optical microscopy (SNOM). In this technique a specimen is scanned with IR beam formed by a fine aperture of subwavelength dimensions, placed in a close proximity from the sample $[28,29]$. The spatial resolution is given approximately by the aperture diameter. To achieve a reasonable resolution, a tapered optical fiber probe with aperture of $10-50 \mathrm{~nm}$ is scanned over surface, without touching it, at a distance of a fraction of wavelength. SNOM can be applied also in the spectroscopic mode, mapping sample at $\lambda$ values where a spectroscopic fingerprint occurs, in absorption, fluorescence or reflection, or even a combination of these quantities. Particularly, SNOM opens up the possibility to get chemical information on a microscopic scale, from cells and their internal structures. It can work in any environment without almost any sample preparation.

The typical transmittance to the IR light of this type of probe is of the order of $10^{-5}$. This strong reduction in intensity due to the fiber aperture is the essential obstacle impossible to avoid with classical tunable light sources. IR FELs provide a way to substantially improve the SNOM technique, boosting its capabilities not only in a static imaging but also by providing the possibility to perform dynamic characterization of ultrafast biological processes.

A risk of damage is particularly restricting factor in probing the biostructures and dynamics of life processes at shorter wavelengths. This is the radiation damage that imposes stringent constraints to irradiation procedures and entails characteristic limitations on application of traditional synchrotron sources to biomedical research. Conventional imaging and diffraction methods cannot achieve atomic resolution on non-repetitive biological structures. Damage that develops during the experiment substantially affects results of most techniques, from radiography to protein imaging and protein diffraction. Classical diffraction approach to structure determination with $\mathrm{SR}$ is loaded with impassable limits. To perform structure analysis a sample of repeatable objects, a crystal, is necessary. Unfortunately, about $40 \%$ of the proteins cannot be crystallized with any of known methods; therefore conventional crystallography is not applicable in this case. Moreover, even crystalline proteins can modify conformation by packing and by unnatural environment that can lead to misinterpretation. Among these extremely difficult to crystallize and purify, are just almost all membrane proteins (MP) that play a key rule in drug transport inside the cells.

A breakthrough in overcoming the restrictions has come at extreme dose rates and ultrashort exposures with advent of new short-wavelength free electron lasers [30]. The new technique is a variant of the coherent X-ray diffraction imaging (CXDI) applicable to non-periodic structures, from single molecules to cells and tissue preparations, at a spatial resolution from subnanometric to micrometric scales, especially important in biological studies [31, 32]. Coherent X-ray diffraction imaging was firstly demonstrated by Miao in 1999 [33], who extended earlier experiments on image reconstruction from diffraction pattern in visible light to X-ray region [34]. First CXDI result of Escherichia coli bacteria stained with $\mathrm{KMnO}_{4}$, obtained with radiation of $\lambda=0.2 \mathrm{~nm}$ at a resolution of $30 \mathrm{~nm}$, has been reported in 2003 [35].

The theory predicts that with an ultrafast, intense and coherent X-ray pulse a diffraction pattern can be recorded from a single object (1-1000 nm), like a molecule, a virus or a cell before the object is damaged and converted into plasma (Neutze [30]). To accomplish this, extremely intense sources are necessary that combine the extreme photon flux in the ultrafast pulses, each containing $10^{12}$ photons or more, excellent coherence, spatial stability and repeatability. This new approach is in fact a form of CXDI with radiation delivered in extremely intense ultrafast pulses. SASE FELs delivering tunable, highly coherent monochromatic radiation in the $\mathrm{XUV}$ to X-ray range of wavelengths are, at present, the only sources suitable to the technique [36]. To achieve the atomic resolution, a pulsed FEL source is necessary, that operates at a wavelength of about $0.1 \mathrm{~nm}$, with pulse duration below 10 fs. The whole pulse energy should be focused on the investigated specimen, that means, in case of single protein diffraction, a need of focusing the beam to $10-100 \mathrm{~nm}$. Real image of the object is derived from diffracted intensity distribution of the beam and with phase retrieval algorithm. The $3 \mathrm{D}$ structure of a protein can be determined in a multishot experiment with $>10^{5}$ shots, each interacting with a new molecule. A train of the molecules is formed with an injector, precisely synchronized with FEL pulses exactly in the beam focus.

After the XUV-FLASH, the prototype of SASE-FEL facility constructed in HASYLAB (Hamburg) has passed successfully examination and confirmed theoretical expectations, the next three devices working in the range of hard X-rays have been designed. In the late 2009 LCLS at SLAC, USA started its operation. Next two will be operational soon: RIKEN XFEL at Harima, Japan (2010), and finally the most advanced construction of the European XFEL at DESY, Germany (2013). The EU-XFEL facility is constructed and will be exploited by international consortium in which also Poland participates [37].

\section{Conclusions}

Free electron lasers are particularly beneficial at biomedical tasks. The new sources span gap between classical lasers and SR synchrotron sources, strongly enhancing probing of soft matter structure and dynamics of life processes with very high spatial and temporal resolution. The new imaging techniques, possible only with 
short-wavelength variants of FEL, open up opportunity to reproduce the soft tissue and proteins with unprecedented spatial resolution. Structural biology will be revolutionized with a unique opportunity of a variant of the CXDI applicable to non-periodic structures, from single molecules to cells and tissue preparations, at a spatial resolution from subnanometric to micrometric scales, especially important in biological studies.

FELs with the tunable, high-peak-power pulsed beams, are capable to produce well-defined photoablative lesions that conform to small, irregularly shaped surgical targets. Investigating with FELs of new medical techniques for surgery and therapy can provide a breakthrough in understanding the ablation mechanisms and lead to new solutions in medical-related issues. The easy FEL tunability will help in solving the problem of tissue inhomogeneity during surgery, that often requires instantaneous modification of source parameters to optimize ablation.

\section{Acknowledgments}

This work has been partially supported by the Ministry of Science and Higher Education of Poland, SPB no. DESY $/ 68 / 2007$.

\section{References}

[1] S. Khan, J. Mod. Opt. 55, 3469 (2008).

[2] V.N. Litvinenko, S.H. Park, I.V. Pinayev, Y. Wu, Nucl. Instrum. Methods Phys. Res. A 475, 195 (2001).

[3] K. Tiedtke, A. Azima, N. von Bargen, L. Bittner, S. Bonfigt, S. Düsterer, B. Faatz, U. Frühling, M. Gensch, Ch. Gerth, N. Guerassimova, U. Hahn, T. Hans, M. Hesse, K. Honkavaar, U. Jastrow, P. Juranic, S. Kapitzki, B. Keitel, T. Kracht, M. Kuhlmann, W.B. Li, M. Martins, T. Nuńez, E. Plönjes, H. Redlin, E.L. Saldin, E.A. Schneidmiller, J.R. Schneider, S. Schreiber, N. Stojanovic, F. Tavella, S. Toleikis, R. Treusch, H. Weigelt, M. Wellhöfer, H. Wabnitz, M.V. Yurkov, J. Feldhaus, New J. Phys. 11, (2009) 023029 (http://iopscience.iop.org/1367-2630/11/2/ 023029).

[4] N. Patel, Nature 415, 110 (2002).

[5] G. Margaritondo, Y. Hwu, J.H. Je, Rivista Nuovo Cimento 27, 1 (2004).

[6] T.L.-M. Sorensen, K.E. McAuley, R. Flaig, E.M.H. Duke, Trends Biotechnol. 24, (2006) 500.

[7] J.B. Pełka, Acta Phys. Pol. A 114, 309 (2008).

[8] G. Wrochna, J. Jagielski, J. Krzywiński, R. Nietubyć, J. Pełka, E. Pławski, J. Sekutowicz, R. Sobierajski, J. Szewiński, Synchrotron Rad. Nat. Sci. 8, 3 (2009).

[9] B. Jean, Nucl. Instrum. Methods Phys. Res. A 393, 540 (1997).

[10] J.I. Youn, P. Sweet, G.M. Peavy, Lasers Surg. Med. 39, 332 (2007).

[11] M. Heya, S. Sano, N. Takagi, Y. Fukami, K. Awazu, Lasers Surg. Med. 32, 349 (2003).
[12] Y. Nakajima, K. Iwatsuki, K. Ishii, S. Suzuki, T. Fuitnaka, T. Yoshimine, K. Awazu, J. Neurosurg. 104, 426 (2006).

[13] M.S. Hutson, B. Ivanov, A. Jayasinghe, G. Adunas, Y.W. Xiao, M.S. Guo, J. Kozub, Opt. Express 17, 9840 (2009).

[14] R.R. Anderson, W. Farinelli, H. Laubach, D, Manstein, A.N, Yaroslavsky, J, Gubeli III, K, Jordan, G.R, Neil, M, Shinn, W, Chandler, G.P, Williams, S, V, Benson, D, R, Douglas, H.F, Dylla, Lasers Surg Med. 38, 913 (2006).

[15] K.M. Joos, L.A. Mawn, J.H. Shen, V.A. Casagrande, Lasers Surg. Med. 32, 32 (2003).

[16] J. Ovelmen-Levitt, K.D. Straub, S. Hauger, E. Szarmes, J. Madey, R.D. Pearlstein, B.S. Nashold, Lasers Surg. Med. 33, 81 (2003).

[17] G.J. Wilmink, S.R. Opalenik, J.T. Beckham, M.A. Mackanos, L.B. Nanney, C.H. Contag, J.M. Davidson, E.D. Jansen, J. Biomed. Optics 13, (2008) 054066.

[18] G. Paltauf, P.E. Dyer, Chem. Rev. (Washington, D.C.) 103, 487 (2003).

[19] A. Vogel, V. Venugopalan, Chem. Rev. (Washington, D.C.) 103, 577 (2003).

[20] V. Venugopalan, A. Guerra, K. Nahen, A. Vogel, Phys. Rev. Lett. 88, 078103 (2002).

[21] M.S. Hutson, X. Ma, Phys. Rev. Lett. 99, 158104 (2007).

[22] G.S. Edwards, M.S. Hutson, J. Synchr. Rad. 10, 354 (2003).

[23] W. Wagner, A. Sokolow, R. Pearlstein, G. Edwards; Appl. Phys. Lett. 94, 013901 (2009).

[24] M.L. Copeland, R.J. Maciunas, G.S. Edwards in: In Neurosurgical Topics: Advanced Techniques in Central Nervous System Metastases, Ed. R.J. Maciunas, The American Association of Neurological Surgeons Park Ridge, IL 1998 Ch. 7.

[25] K.M. Joos, J.H. Shen, D.J. Shetlar, V.A. Casagrande Lasers Surg. Med. 27, 191 (2000).

[26] G.S. Edwards, R.H. Austin, F.E. Carroll, M.L. Copeland, M.E. Couprie, W.E. Gabella, R.F. Haglund, B.A. Hooper, M.S. Hutson, E.D. Jansen, K.M. Joos, D.P. Kiehart, I. Lindau, J. Miao, H.S. Pratisto, J.H. Shen, Y. Tokutake, A.F.G. van der Meer, A. Xie, Rev. Sci. Instrum. $\mathbf{7 4}$ 3207 (2003).

[27] V.N. Litvinenko, S.H. Park, I.V. Pinayev, Y. Wu, M. Emamian, N. Hower, P. Morcombe, O. Oakeley, G. Swift, P. Wang, Nucl. Instrum. Methods Phys.Res. A 429, 151 (1999).

[28] A. Cricenti, J. Alloys Comp. 328, 2 (2001).

[29] A. Cricenti, R. Generosi, P. Perfetti, J.M. Gilligan, N.H. Tolk, C. Coluzza, G. Margaritondo, Appl. Phys. Lett. 73, 151 (1998).

[30] R. Neutze, R. Wouts, D, van der Spoel, E. Weckert, J. Hajdu, Nature 406, 752 (2000).

[31] R. Neutze, G. Huldt, J. Hajdu, D. van der Spoel, Radiat. Phys. Chem. 71, 905 (2004). 
[32] H.N. Chapman, A. Barty, M.J. Bogan, S, Boutet, M, Frank, S.P, Hau-Riege, S, Marchesini, B.W, Woods, S, Bajt, W.H, Benner, R.A, London, E, Plönjes, M, Kuhlmann, R.Treusch, S, Düsterer, T, Tschentscher, J.R, Schneider, E, Spiller, T, Möller, C, Bostedt, M, Hoener, D.A, Shapiro, K.O, Hodgson, D, van der Spoel, F.Burmeister, M, Bergh, C, Caleman, G, Huldt, M, M, Seibert, F.R.N.C, Maia, R.W, Lee, A, Szöke, N, Timneanu, J, Hajdu, Nature Physics 2, 839 (2006).
[33] J. Miao, P. Charalambous, J. Kirz, D. Sayre, Nature 400, 342 (1999).

[34] J.R. Fienup, J. Opt. Soc. Am. A 4, 118 (1987).

[35] J. Miao, K.O. Hodgson, T. Ishikawa, C.A. Larabell, M.A. LeGros, Y. Nishino, Proc. Natl. Acad. Sci. 100, 110 (2003).

[36] R. Bonifacio, C. Pellegrini, L.M. Narduci, Opt. Commun. 50, 373 (1984).

[37] http://www.xfel.eu/en/. 